\title{
Treatment Response on Positive Symptoms of Schizophrenia with Olanzapine and Risperidone
}

\author{
Khan FZ1 ${ }^{1}$, Rashid ${ }^{2}$, Sultana $\mathrm{SP}^{3}$, Rahman $\mathrm{W}^{4}$, Nazrina $\mathrm{S}^{5}$, Ara $\mathrm{H}^{6}$ \\ DOI: https://doi.org/10.3329/jafmc.v15i1.48644
}

\begin{abstract}
Introduction: Positive symptoms become perceptible in patients as schizophrenia progresses. If schizophrenia goes untreated catatonic motor behaviors are develops. Now a day's catatonia which is a type of disturbed behavior is observed, with less frequency than in past years due to advances in treating schizophrenia. Treatment of schizophrenia by olanzapine and risperidone shows diverse outcomes.
\end{abstract}

Objectives: To assess the response on positive symptoms of schizophrenia by olanzapine and risperidone.

Materials and Methods: This study was conducted in Department of Pharmacology and Department of Psychiatry of Bangabandhu Sheikh Mujib Medical University (BSMMU), Dhaka during the period from September 2013 to January 2015. A total of 179 schizophrenia patients fulfilling the inclusion criteria were enrolled and of them 75 patients completed the study, where 40 were in olanzapine treated group and 35 were in risperidone treated group in a 12-weeks assessment where Scale for Assessment of Positive Symptoms (SAPS) was applied. After statistical analysis of scores between intergroup and intra group $p<0.05$ was considered significant.

Results: Treatment with both the olanzapine and risperidone were remarkably effective for progress of positive symptoms of schizophrenia patients but in olanzapine treated group the score was reduced more than that of risperidone treated group and the difference was significant $(p<0.05)$.

Conclusion: Olanzapine has responded better on positive symptoms of schizophrenia patients than that of risperidone.

Key-words: Schizophrenia, Positive Symptoms, Olanzapine, Risperidone.

\section{Introduction}

Now a day's, schizophrenia is well known psychiatric disorder for its chronic recurrences ${ }^{1}$. Schizophrenia patients may present with positive and/or negative symptoms. The positive symptoms represents with distortion or exaggeration of functions that are normally present, whereas negative symptoms represents with deficiency of mental functions that are normally present. Positive symptoms include hallucinations, delusions, bizarre or disorganized behavior. Disorganized thinking and behavior becomes apparent in patients' speech patterns as schizophrenia progresses and in severe cases patients interpret reality abnormally. Usually in initial state of psychosis patient develops positive symptoms and if treated early the prognosis is better. If untreated or improper treatment the negative symptoms may start to develop. After developing negative symptoms the prognosis is poor than that of positive symptom patients. With this treatment about $30-40 \%$ patients may show signs of poor response to conventional antipsychotics ${ }^{2}$ and among them approximately $50 \%$ may suffer from serious side effects ${ }^{3}$. Therefore the aim of new drug development for schizophrenia treatment has shifted to synthesize compounds capable to improve both positive and negative symptoms, which are commonly insensitive to classical antipsychotics and to synthesize compounds that create slight or no extrapyramidal side effects. Initially, first generation antipsychotics seemed to be more efficient than second generation but later by meta-analysis of effects of first generation antipsychotics with second generation, the first generation drugs were found to be more effective in schizophrenic patients ${ }^{4}$. Olanzapine is a thienobenzo-diazepine and has high affinity to serotonin, histamine, a1adrenergic and dopamine receptors ${ }^{5,6}$. It has shown that olanzapine has better efficacy and less side effects compared to haloperidol in controlled clinical trials and some studies reveals that it has better efficacy in schizophrenia patient management ${ }^{7,8,9}$. Risperidone is a benzisoxazole derivative and has greatest affinity for serotonin, histamine, $\alpha 1$ adrenerigic and dopamine sites. In few other trials, risperidone has been shown to be better to typical antipsychotics?

In the treatment of psychotic disorders both the risperidone and olanzapine have shown well tolerability and efficacy $y^{6,7,9,10}$. A variety of study for comparing these two atypical antipsychotics has resulted in various outcomes. For example, Tran et al., and Gureje et al had found olanzapine to have a risk vs benefit advantage compared to risperidone ${ }^{10,11}$. In a study, advantage of olanzapine was due to its better efficacy and improvement of symptoms in older patients ${ }^{10}$. But symptoms, better maintenance of treatment and finally lower incidence of extrapyramidal side effects, hyperprolactinemia and sexual dysfunction ${ }^{10}$. Also, Edgell et $\mathrm{al}^{12}$ and Rascati et $\mathrm{al}^{13}$ found that olanzapine treated patients were more likely to continue treatment than risperidone treated patients. Feldman et $\mathrm{al}^{14}$ also, found olanzapine to be more efficacious than risperidone in progress of older patients. On the other hand, in parallel comparisons, Taylor et $a{ }^{15}$ Kasper et $\mathrm{a}^{16}$ and Conley and Mahmoud ${ }^{17}$, found equal clinical outcome for both of olanzapine and risperidone treated patients. Based on aforesaid controversies and result, in present study, the efficacy on positive symptoms of schizophrenia of risperidone and olanzapine were compared for additional persuasive evidence.

1. Lt Col Fatema Zerin Khan, MBBS, FCGP, MD, Commanding Officer, 71 field Ambulance, Jashore (Email: majorfatema@gmail. com) 2. Dr Nafisa Rashid, MBBS, MPhil, Assistant Professor of Pharmacology, Addin Women's Medical College, Dhaka 3. Dr Syeda Papia Sultana, MBBS, MD, Assistant Professor of Pharmacology, Ashiyan Medical College, Dhaka 4. Lt Col Wahida Rahman, MBBS, MPhil, Associate Professor of Pharmacology, Armed Forces Medical College (AFMC), Dhaka 5. Lt Col Sayeda Nazrina, MBBS, FCGP, MPhil, Associate Professor of Pharmacology, AFMC, Dhaka 6. Dr Hosnea Ara, MBBS, MD, Assistant Professor of Psychiatry, Addin Women's Medical College, Dhaka. 


\section{Materials and Methods}

Present study was carried out in Department of Pharmacology and Department of Psychiatry of BSMMU during the period from September 2013 to January 2015. Samples were collected up to November 2014. Patients with schizophrenia attending the outpatient department or admitted in the inpatient department of psychiatry, BSMMU were recruited in the study. Patients were diagnosed as schizophrenic, by psychiatrists of BSMMU according to diagnostic protocol which fulfilled DSM-5 criteria (Diagnostic and Statistical Manual of Mental Disorders, 2013). Patients receiving antipsychotic drugs within last 30 days, having serious cognitive deficit and having serious infection or terminal illness were excluded from the study. A total of 179 patients fulfilling the inclusion criteria were enrolled and of them 75 patients completed the study, where 40 were in olanzapine treated group and 35 were in risperidone treated group. In this 12 weeks study the patients were assessed by means of SAPS at baseline and at 4,8 and 12 weeks. Olanzapine and risperidone were prescribed according to practice guidelines at a dose of 5-20 mg/day and 4-16 mg/day orally respectively. Data were collected on variables of interest using a semi-structured questionnaire (research instrument) by interviewing. Collected data was checked daily and edited into Excel sheet. Data were processed and analyzed using computer software SPSS (Statistical Package for Social Sciences) version 16.0. Patients were compared on baseline characteristics by means of 't'-tests. Treatment efficacy was analyzed by unpaired 't'-test and paired 't'-test was used to compare the continuous data between and within groups respectively. The level of significance was set at $5 \%$ and $p<0.05$ was considered significant.

\section{Results}

Table-I shows, in olanzapine and risperidone treated groups at baseline mean \pm SD of SAPS score were $62.51 \pm 3.06$ and $61.11 \pm 3.45$ respectively. The difference in SAPS score between olanzapine and risperidone treated group at baseline was not significant $(p>0.05)$. Table-Il shows, in olanzapine treated group SAPS score was reduced from $62.51 \pm 3.06$ to $58.1 \pm 5.5$, $49.5 \pm 5.5,40.5 \pm 6.3$ after 4,8 and 12 weeks of drug treatment respectively. Whereas in risperidone treated group SAPS score was reduced from $61.11 \pm 3.45$ to $59.9 \pm 4.9,51.7 \pm 4.4$ and $45.7 \pm 5.5$ after 4,8 and 12 weeks of drug treatment respectively. Table-III shows, in olanzapine treated group SAPS score was reduced from $62.51 \pm 3.06$ to $40.5 \pm 6.3$ at 12 weeks of drug treatment and the reduction was significant $(p<0.05)$. Whereas in risperidone treated group BPRS score was reduced from $61.11 \pm 3.45$ to $45.7 \pm 5.5$ and the reduction was also significant $(p<0.05)$. Table-IV shows, in olanzapine treated group the score was reduced more than that of risperidone treated group and the difference was significant $(p<0.05)$.

Table-I: SAPS scores of study group at baseline

\begin{tabular}{|l|c|c|c|}
\hline & \multicolumn{2}{|c|}{ Group } & \multirow{2}{*}{$\mathrm{p}$-value } \\
\cline { 2 - 3 } & Olanzapine( $\mathrm{n}=40)$ & Risperidone( $\mathrm{n}=35)$ & \\
\hline SAPS scores & $62.51 \pm 3.06$ & $61.11 \pm 3.45$ & $>0.05$ \\
\hline
\end{tabular}

- Data were presented as mean $\pm S D$ and were analyzed by unpaired 't'test
Table-II: SAPS scores at baseline, 4 weeks, 8 weeks and 12 weeks of treatment

\begin{tabular}{|l|c|c|c|c|}
\hline \multicolumn{1}{|c|}{ Groups } & At Baseline & At 4th week & At 8th week & At 12 weeks \\
\hline $\begin{array}{l}\text { Olanzapine } \\
\text { treated group } \\
(n=40)\end{array}$ & $62.51 \pm 3.06$ & $58.1 \pm 5.5$ & $49.5 \pm 5.5$ & $40.5 \pm 6.3$ \\
\hline $\begin{array}{l}\text { Risperidone } \\
\text { treated group } \\
(n=35)\end{array}$ & $61.11 \pm 3.45$ & $59.9 \pm 4.9$ & $51.7 \pm 4.4$ & $45.7 \pm 5.5$ \\
\hline
\end{tabular}

- Data were presented as mean $\pm S D$

Table-III: SAPS scores at baseline and 12 weeks of treatment

\begin{tabular}{|l|c|c|c|}
\hline \multicolumn{1}{|c|}{ Group } & At Baseline & At 12 weeks & p-value \\
\hline Olanzapine treated group $(n=40)$ & $62.51 \pm 3.06$ & $40.5 \pm 6.3$ & $<0.0001$ \\
\hline Risperidone treated group $(n=35)$ & $61.11 \pm 3.45$ & $45.7 \pm 5.5$ & $<0.0001$ \\
\hline
\end{tabular}

- Data were presented as mean $\pm S D$ and were analyzed using Paired t-Test

Table-IV: Comparison of SAPS scores between olanzapine and risperidone groups after 12 weeks of treatment

\begin{tabular}{|l|c|c|c|}
\hline & \multicolumn{2}{|c|}{ Group } & \multirow{2}{*}{-value } \\
\cline { 2 - 3 } & Olanzapine $(\mathrm{n}=40)$ & Risperidone $(\mathrm{n}=\mathbf{3 5})$ & \\
\hline SAPS scores & $40.5 \pm 6.3$ & $45.7 \pm 5.5$ & $<0.001$ \\
\hline
\end{tabular}

- Data were presented as mean $\pm S D$ and were analyzed using unpaired t-Test

\section{Discussion}

The prime intention of this study was to evaluate once more the safety and effectiveness of olanzapine and risperidone in treatment of schizophrenic patients. Ultimately the outcomes of both olanzapine and risperidone were appreciably effective in reducing the overall psychotic symptoms. Nevertheless, the findings are a bit comparable to a different analogous double-blind, 28 weeks study on 339 patients meeting DSM-IV criteria for schizophrenia, schizophreniform or schizoaffective disorder ${ }^{10}$. In current appraisal there was no significant variation amongst olanzapine and risperidone in respect to association of positive symptoms. There are few other clinical trials having varying outcomes; such as, in a head to head clinical trial for 8 weeks comparing olanzapine with risperidone on patients meeting DSM-IV criteria for schizophrenia or schizoaffective disorder, no significant variation found among them with respect to efficacy or side effects ${ }^{17}$, in existing consideration significant deterioration of adverse effects was apparent only by risperidone. Another study of olanzapine vs risperidone concluded that, though there was better efficacy in risperidone group, there was considerable raise in side effects as well in the same group compared to olanzapine treated group ${ }^{18}$. A study by Robinson et a ${ }^{19}$ evaluated comparison of 4 months treatment outcomes for olanzapine vs risperidone in patients with first episode schizophrenia spectrum disorders. Among one hundred twelve subjects there were with first episode schizophrenia, schizophreniform or schizoaffective disorder which were randomly assigned to treatment with olanzapine (2.5-20 mg/day) or risperidone (1-6 mg/ day). At the end of the study clinical outcomes with risperidone were equal to those with olanzapine. A prospective observational 
study by Ascher-Svanum et al olanzapine and risperidone treaded group were compared one year for assessing rate, duration and time to hospitalization. In this stud,y 516 patients who were newly initiated on olanzapine or risperidone were recruited, a total of 271 patients comprising the olanzapine $(n=159)$ and risperidone $(n=112)$ treatment groups. Compared to risperidone, olanzapine treated patients had lesser hospitalization rates $(24.1 \%$ vs $14.4 \%$ respectively) and smaller number hospitalization days (14.5 days vs 9.9 days respectively).

Similarly, there are other studies that have similar statement in support of risperidone ${ }^{14,15}$. Possibly, applying different efficacy measures with various psychomotor properties and different samples using distinct diagnostic criteria may elucidate these contradictory results to some extent. In addition, as weight gain rate was noticeably higher in the olanzapine group, it became an important trouble especially for women, and such metabolic side effect, should always be taken in consideration by treating clinician. Equal vigilance also should be applied regarding extrapyramidal symptoms which are more challenging with risperidone. In a Cochrane review article by Komossa et $\mathrm{al}^{20}$ risperidone improved the general mental state (PANSS total score) slightly less than olanzapine but slightly more than quetiapine and ziprasidone. Whilst in comparison with the earlier studies, the result of the present study may not be shown to be more influential and free from controversy. It may put forward the requisite of more analogous trials regarding comparison between atypical antipsychotics. Instead of diagnosing whole of schizophrenia, schizoaffective and schizophreniform disorders like aforesaid trials, restriction to only schizophrenia diagnosis, could support a more accurate trial in the present assessment. Moreover, equal efficacy of these two second generation antipsychotics in progress of positive symptoms may weaken any kind of irrational clinical preference during selection one of them as first choice. The number of patients studied was not large enough to draw a definitive conclusion and short duration of assessment, gender, habitat, lack of placebo arm may have radically impact on the evaluate sensitivity to the study. Thus the discrepancy of our results with other studies could be due to those factors. Further comparable trials are expected in upcoming days to improve our knowledge in this regard.

\section{Conclusion}

Even though both of olanzapine and risperidone are similarly efficient for the treatment of schizophrenia, in present study olanzapine showed better response with respect to positive symptoms.

\section{References}

1. Kane JM. Schizophrenia. The New England Journal of Medicine 1996; 334(1):34-41.

2. Lieberman JA. Prediction of outcome in first-episode schizophrenia. Journal of Clinical Psychiatry 1993; 54(3):13-7.

3. Kane JM, Lieberman JA. Adverse Effects of Psychotropic Drugs. New York: Guilford Press; 1992.

4. Leucht S, Corves C, Arbter D et al. Second-generation versus firstgeneration antipsychotic drugs for schizophrenia: A meta-analysis. The Lancet 2009; 373(9657):31-41.

5. Moore NA, Tye NC, Axton MS et al. The behavioral pharmacology of olanzapine, a novel "atypical" antipsychotic agent. Journal of Pharmacology and Experimental Therapeutics 1992; 262(2):545-51.

6. Beasley Jr CM, Tollefson G, Tran P et al. Olanzapine versus placebo and haloperidol: Acute phase results of the North American Double-Blind Olanzapine Trial. Neuro Psycho Pharmacology 1996; 14(2):111-23.

7. Tollefson GD, Beasley Jr CM, Tran PV et al. Olanzapine versus haloperidol in the treatment of schizophrenia and schizoaffective disorders: results of an international collaborative trial. The American Journal of Psychiatry 1997; 154:457-65.

8. Dellva MA, Tran P, Tollefson GD et al. Standard olanzapine versus placebo and ineffective-dose olanzapine in the maintenance treatment of schizophrenia. Psychiatric Services 1997; 48(12):1571-7.

9. Marder SR, Meibach RC. Risperidone in the treatment of schizophrenia. American Journal of Psychiatry 1994; 151(6):825-35.

10. Tran PV, Hamilton SH, Kuntz AJ et al. Double-blind comparison of olanzapine versus risperidone in the treatment of schizophrenia and other psychotic disorders. Journal of Clinical Psychopharmacology 1997; 17(5):407-18.

11. Gureje O, Miles W, Keks N et al. Olanzapine vs risperidone in the management of schizophrenia: A randomized double-blind trial in Australia and New Zealand. Schizophrenia Research 2003; 61(2-3):303-14.

12. Edgell ET, Andersen SW, Johnstone BM et al. Olanzapine versus risperidone: A prospective comparison of clinical and economic outcomes in schizophrenia. Pharmaco Economics 2000; 18(6):567-79.

13. Rascati $\mathrm{KL}$, Johnsrud MT, Crismon ML et al. Olanzapine versus risperidone in the treatment of schizophrenia: a comparison of costs among Texas Medicaid recipients. Pharmaco Economics 2003; 21(10):683-97.

14. Feldman PD, Kaiser CJ, Kennedy JS et al. Comparison of risperidone and olanzapine in the control of negative symptoms of chronic schizophrenia and related psychotic disorders in patients aged 50 to 65 years. The Journal of Clinical Psychiatry 2003; 64(9):998-1004.

15. Taylor DM, Wright T, Libretto SE. Risperidone compared with olanzapine in a naturalistic clinical study: A cost analysis. Journal of Clinical Psychiatry 2003; 64(5):589-97.

16. Kasper S, Jones M, Duchesne I. Risperidone olanzapine drug outcomes studies in Schizophrenia (RODOS): Health economic results of an international naturalistic study. International Clinical Psychopharmacology 2001; 16(4):189-96.

17. Conley RR, Mahmoud R. A randomized double-blind study of risperidone and olanzapine in the treatment of schizophrenia or schizoaffective disorder. The American Journal of Psychiatry 2001; 158(5):765-74.

18. Ho BC, Miller D, Nopoulos P, Andreasen NC. A comparative effectiveness study of risperidone and olanzapine in the treatment of schizophrenia. Journal of Clinical Psychiatry 1999; 60(10):658-63.

19. Robinson DG, Woerner MG, Napolitano B et al. Randomized comparison of olanzapine versus risperidone for the treatment of first-episode schizophrenia: 4-month outcomes. American Journal of Psychiatry 2006; 163(12):2096-102.

20. Komossa K, Rummel-Kluge R, Schwarz S et al. Risperidone versus other atypical antipsychotics for Schizophrenia. Cochrane Database of Systemic Review 2011. 